\title{
Carbon Nanotube Millikelvin Transport and Nanomechanics
}

\author{
K. J. G. Götz, ${ }^{1}$ F. J. Schupp, ${ }^{1,2}$ and A. K. Hüttel ${ }^{1}$ \\ ${ }^{1}$ Institute for Experimental and Applied Physics, University of Regensburg, Universitätsstraße 31, 93053 Regensburg, Germany \\ ${ }^{2}$ Department of Physics, Princeton University, Princeton, New Jersey 08544, USA
}

\begin{abstract}
Single wall carbon nanotubes cooled to cryogenic temperatures are outstanding electronic as well as nanoelectromechanical model systems. To probe a largely unperturbed system, we measure a suspended carbonnanotube device where the nanotube is grown in the last fabrication step, thus avoiding damage and residues from subsequent processing. In this ultra-clean device, we observe the transport spectrum and its interaction with nano-electromechanics over a wide gate voltage range and thereby over a wide range of coupling parameters between the quantum dot and the contact electrodes.
\end{abstract}

\section{INTRODUCTION}

Both the electronic and the nano-electromechanical lowtemperature properties of single-wall carbon nanotubes have attracted significant research over the past decades, resulting in a large spectrum of publications [1]. In particular "ultraclean", as grown carbon nanotubes are prototypical singleelectron devices [2], where the unperturbed transport spectrum reveals a wide range of phenomena originating from the band structure [3-6] and the interaction between the charge carriers [7-10]. At the same time, single-wall carbon nanotubes at millikelvin temperatures are excellent mechanical resonators [11-13], with resonance frequencies up to $39 \mathrm{GHz}$ [14] and quality factors up to $5 \times 10^{6}$ [15]. In suspended carbon nanotube devices, the electrostatic forces acting on single electrons dominate the mechanical behaviour, and electronic transport is subject to strong coupling between single electron tunneling and vibrational motion [16-18].

Typically, reports focus on selected aspects of the behaviour of carbon nanotubes, possibly across several devices. While this is a suitable approach for in-depth analysis of the observed phenomena, it is challenging to obtain a complete picture of the behaviour of one single wall nanotube device across all regimes of coupling to the leads. Here, we present low-temperature measurement data collected on a single device. We follow both electronic and nano-electromechanical phenomena from the highly transparent transport through hole states, across the band gap to strong Coulomb blockade, and finally towards larger electron numbers, where the tunnel barriers become increasingly transparent with higher order transport processes dominating the current.

The schematic device geometry is sketched in Fig. 1(a). The device fabrication follows the approach of [2]. We start with a highly p++ doped silicon wafer having a 500nm drygrown thermal surface oxide. The contact electrodes, with a bilayer of $10 \mathrm{~nm}$ tungsten and $40 \mathrm{~nm}$ platinum, are fabricated using electron beam lithography, metal evaporation, and liftoff. Subsequently, the silicon oxide surface is anisotropically etched by $100 \mathrm{~nm}$ to deepen the trenches between the electrodes. Nanotube growth catalyst is locally deposited via an additional lithography step, drop-casting, and lift-off [19]. In the final fabrication step, the carbon nanotubes are grown in situ by chemical vapour deposition [19]. The distance be-
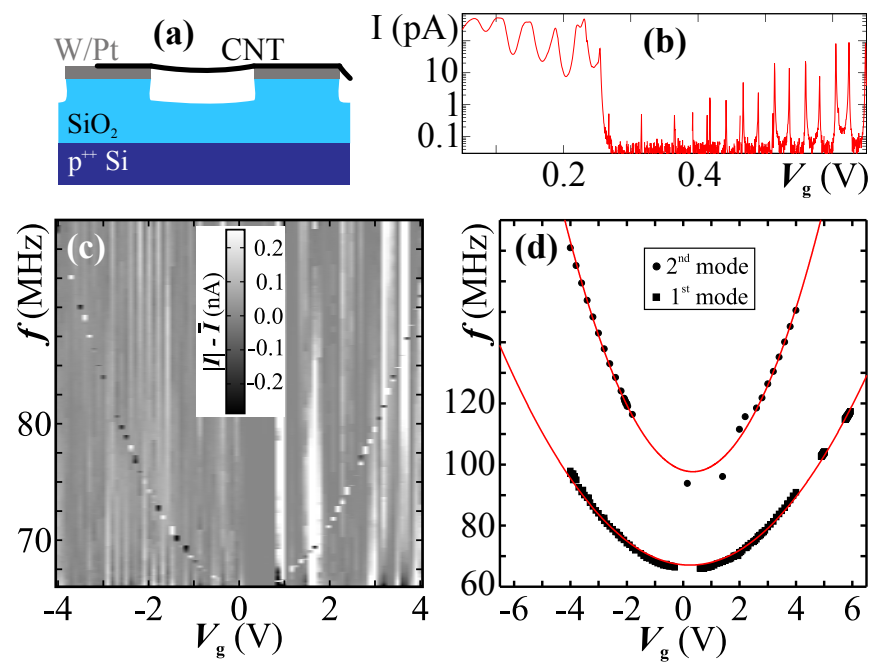

FIG. 1. (a) Sketch of the geometry of the measured device. (b) Current $I\left(V_{\mathrm{g}}\right)$ at $V_{\mathrm{sd}}=20 \mu \mathrm{V}$ around the electronic band gap. Hole transport for $V_{\mathrm{g}}<0.3 \mathrm{~V}$, band gap, and electron transport for $V_{\mathrm{g}}>0.3 \mathrm{~V}$ with an electronic shell pattern can be clearly discerned. (c) Measured dc current as function of gate voltage $V_{\mathrm{g}}$ and driving rf frequency $f, I\left(f, V_{\mathrm{g}}\right)-\bar{I}\left(V_{\mathrm{g}}\right)$, with the average current per frequency trace subtracted. The transversal vibration resonance becomes visible as a sequence of peaks or dips [13]. $V_{\mathrm{sd}}=-0.1 \mathrm{mV}$, nominal rf generator power $P=2 \mathrm{dBm}$. (d) Extracted resonance frequency $f_{0}\left(V_{\mathrm{g}}\right)$ for the fundamental vibration mode of (c) and a higher mode; the solid lines are parabolic fits.

tween the contact electrodes is $L=1.2 \mu \mathrm{m}$, providing a lower boundary for the length of the active nanotube segment.

A measurement of the low-bias $\left(V_{\mathrm{sd}}=20 \mu \mathrm{V}\right)$ current through the device at base temperature $T \leq 25 \mathrm{mK}$ of the dilution refrigerator is plotted in Fig. 1(b). On the hole conduction side, i.e. for $V_{\mathrm{g}}<0.3 \mathrm{~V}$, the device is highly transparent, while the electron conduction side, $V_{\mathrm{g}}>0.3 \mathrm{~V}$, initially displays strong Coulomb blockade. This indicates that the actual contacts between nanotube and platinum electrodes are transparent. The opaque tunnel barriers of the quantum dot at low electron number are given by the extended $\mathrm{p}-\mathrm{n}$ junctions between the electrostatically $n$-doped central nanotube segment and the nanotube segments close to the leads [3, 20,21].

Figure 1(c) shows a measurement of the transversal vibrational resonance of the carbon nanotube $[11,12]$. Here, the 

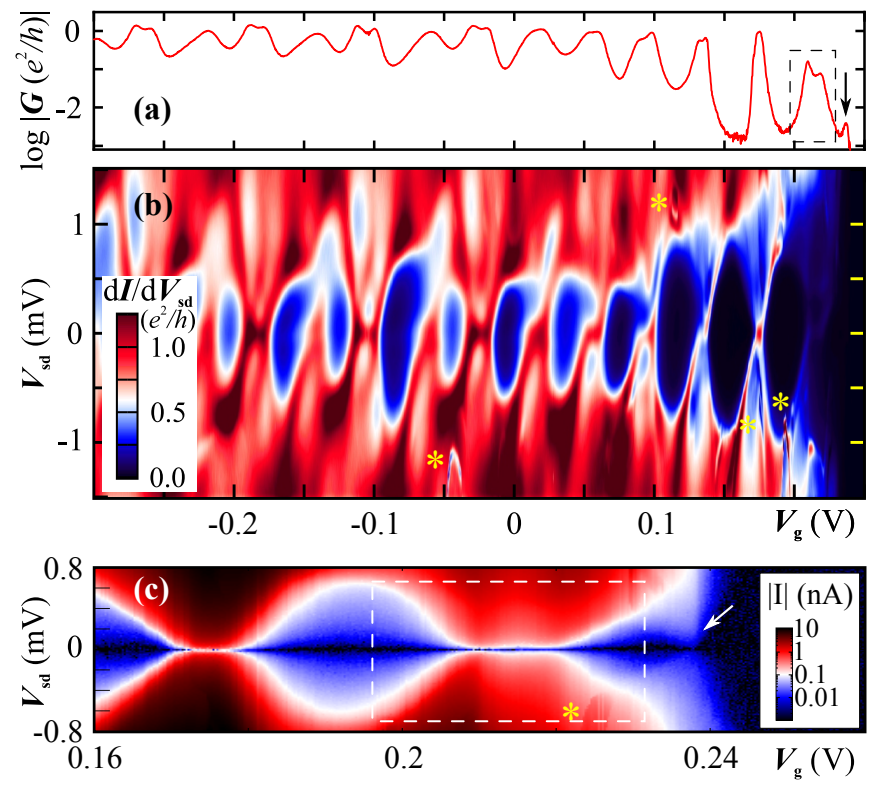

FIG. 2. Transport spectrum of the few-hole parameter region of the device: (a) zero-bias differential conductance $\mathrm{d} I / \mathrm{d} V_{\mathrm{sd}}\left(V_{\mathrm{g}}\right)$ and (b) stability diagram $\mathrm{d} I / \mathrm{d} V_{\mathrm{sd}}\left(V_{\mathrm{g}}, V_{\mathrm{sd}}\right)$ at identical $V_{\mathrm{g}}$-axis. Zero bias ridges of enhanced conductance indicate the presence of the Kondo effect. (c) Detail zoom of the band gap edge, now plotting the absolute value of the measured current $\left|I\left(V_{\mathrm{g}}\right)\right|$. Yellow asterisks mark mechanical instability features, see the text.

detection scheme of $[13,16,22]$ is used: an antenna several millimeters from the device radiatively introduces a $\mathrm{MHz}$ signal, and the resulting driven vibration at mechanical resonance leads to a peak or dip in detected dc-current due to the modulated displacement of the nanotube and the resulting modulation in gate capacitance. We observe a strong gate voltage dependence of the resonance frequency $f_{0}\left(V_{\mathrm{g}}\right)$ due to the changing tension in the nanotube induced by the pull of the gate voltage. The resonance from Fig. 1(c) and an additional second mode at higher frequency are extracted in Fig. 1(d). In both cases, a parabolic fit provides a good (though strongly simplified) approximation of the gate voltage dependence in the measured range (cf. [12, 23] for a more detailed model).

\section{HOLE TRANSPORT REGION}

At gate voltages $V_{\mathrm{g}}<0.3 \mathrm{~V}$, current is carried by valence band states, and the quantum dot is charged with an increasing number of holes for decreasing gate voltage. Due to the absence of abovementioned p-n barriers, the device displays strong coupling to the metal contacts. This is illustrated in Fig. 2; note that while the line plot of Fig. 2(a) is logarithmic, the color scale of Fig. 2(b) is linear. The pronounced sequence of conductance oscillations at zero bias turns, upon closer observation, out to be a sequence of Kondo conductance ridges $[6,24-26]$ corresponding each to the addition of two holes. This is particluarly obvious around $V_{\mathrm{g}}=-0.03 \mathrm{~V},-0.11 \mathrm{~V}$,
$-0.19 \mathrm{~V}$, where a clear Kondo zero bias anomaly of conductance emerges. The yellow asterisks in Fig. 2 mark characteristic lobe-shaped features with sharp edges in the data which correspond to mechanical instability and vibrational feedback phenomena in transport [16, 22, 27, 28].

Figure 2(c) zooms in on the band gap edge; here we plot the absolute value of the dc current $\left|I\left(V_{\mathrm{g}}, V_{\mathrm{sd}}\right)\right|$ in logarithmic scale since this allows us to resolve smaller signals. The feature at $V_{\mathrm{g}}=0.22 \mathrm{~V}$ turns out to be a clear double-peak, i.e., two nearly merged Coulomb oscillations, consistent with the curve shape of Fig. 2(a) [see the dashed boxes in both figures]. Since the Kondo ridges are associated with odd electron or hole numbers, this means that at $V_{\mathrm{g}} \approx 0.23 \mathrm{~V}$ the nanotube is charged with an even number of holes. This leaves us with a conundrum about the nature of the structure at $V_{\mathrm{g}} \approx 0.24 \mathrm{~V}$, also visible as a single sharp peak in Fig. 2(a) and marked in both figures with an arrow. Two explanations are possible: either this is a single Coulomb oscillation and an additional oscillation cannot be resolved due to too small tunnel rates, or this peak is already a merger of two oscillations. The former explanation seems unlikely since, even at high bias, no further structures in current or conductance are visible at the hole side of the band gap region. Measurements at larger temperature and at finite magnetic field may provide additional information.

A stability diagram at larger hole number, where the interfaces between the carbon nanotube and its contact electrodes become increasingly transparent towards Fabry-Pérot conduction [31, 32], is plotted in Fig. 3(a). In absence of the (SU(2)) Kondo effect at even electron number, elastic co-tunneling enables large conductance in nominal Coulomb blockade regions [33]. For odd electron numbers, Coulomb blockade is suppressed at low bias, and, as already observed in Fig. 2, several of the conductance peaks merge completely into Kondo ridges. This is also clearly visible in the line plot of Fig. 3(b), displaying the current at $V_{\mathrm{sd}}=-0.1 \mathrm{mV}$.

The subseqent panel, Fig. 3(c), shows the result of mechanical resonance detection in the same gate voltage region, using the same measurement and plotting scheme as Fig. 1(c), though at much lower driving power (nominally $P=-13.7 \mathrm{dBm}$ ). The fundamental vibration mode becomes clearly visible as a diagonal feature. In the plotted gate voltage region it displays a near-linear dependence on the gate voltage $V_{\mathrm{g}}$, qualitatively different from the observations of, e.g., $[16,30,34,35]$, where each Coulomb oscillation causes a strong dip in frequency due to changing electrostatic forces in response to single charge tunneling at the charge degeneracy points. This indicates that, due to increased tunnel rates and lifetime broadening of the involved quantum levels, Coulomb blockade is gradually lost [29] and that the nanotube more and more resembles a metallic beam. However, a more detailed analysis (Fig. 3(d)), where the resonance frequency $f_{0}\left(V_{\mathrm{g}}\right)$ has been extracted and a linear contribution has been removed, still allows to identify some of the characteristic oscillations of $\tilde{\omega}_{0}\left(V_{\mathrm{g}}\right)$, corresponding to the addition of single holes to the system [16, 29]; selected features are marked in the figure 

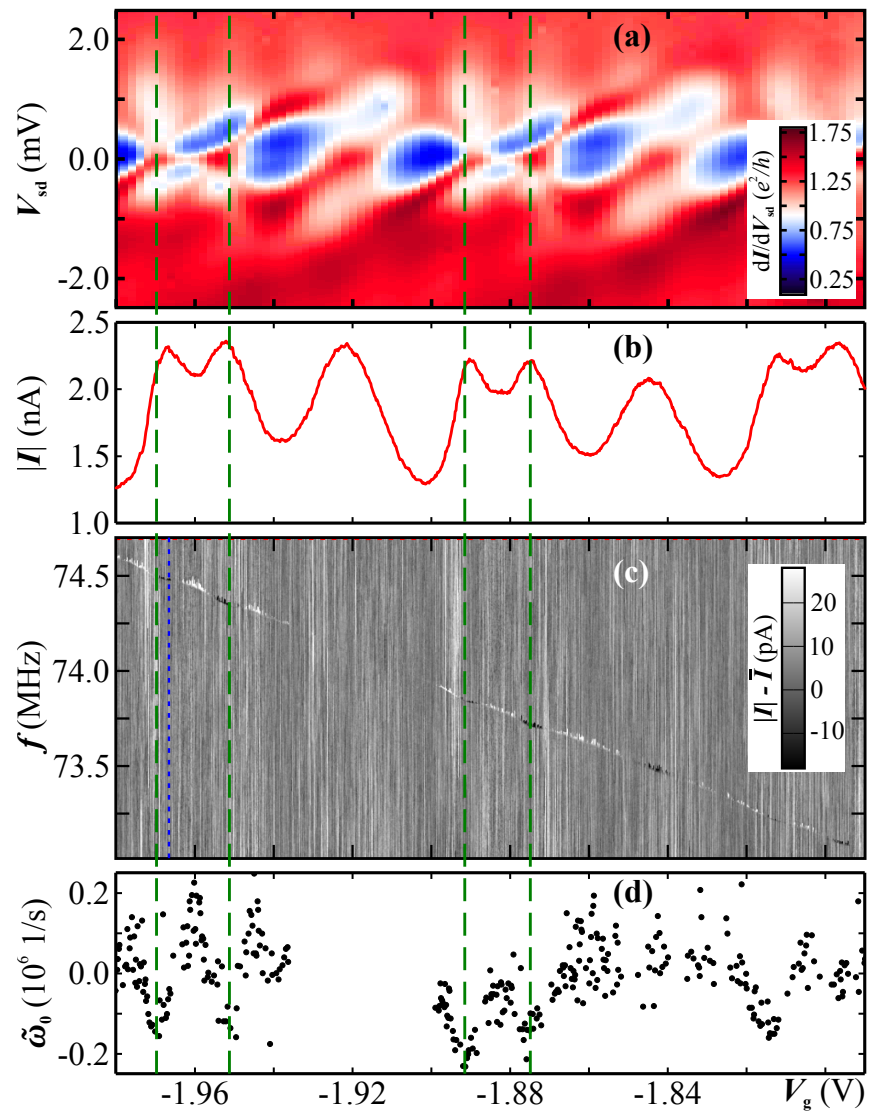

FIG. 3. Behaviour at large hole number, in the transition region between Kondo transport and Fabry-Pérot interference. (a) Stability diagram showing $\mathrm{d} I / \mathrm{d} V_{\mathrm{sd}}\left(V_{\mathrm{g}}, V_{\mathrm{sd}}\right)$. Again zero-bias ridges of enhanced conductance clearly indicate the Kondo effect. (b)-(d) Mechanical resonance frequency detection in the same gate voltage region: (b) off-resonant current $I\left(V_{\mathrm{g}}\right)$ for $V_{\mathrm{sd}}=-0.1 \mathrm{mV}$, (c) current as function of gate voltage $V_{\mathrm{g}}$ and driving rf frequency $f, I\left(f, V_{\mathrm{g}}\right)-\bar{I}\left(V_{\mathrm{g}}\right)$, with the average current per frequency trace subtracted; $V_{\mathrm{sd}}=-0.1 \mathrm{mV}$, nominal rf driving signal power $P=-13.7 \mathrm{dBm}$. (d) Resonance (angular) frequency $\tilde{\omega}_{0}\left(V_{\mathrm{g}}\right)=2 \pi f_{0}\left(V_{\mathrm{g}}\right)-\left(a V_{\mathrm{g}}+b\right)$ extracted from (c), with a term linear in $V_{\mathrm{g}}$ subtracted; see also [16, 29, 30].

with vertical dashed lines.

For even larger negative gate voltages, our device unambiguously enters the Fabry-Pérot regime, where weak scattering of the electronic wavefunctions at the contacts causes quantum interference [31]. A detailed discussion of the properties of the device in this parameter region can be found in [32].

\section{STRONG COULOMB BLOCKADE}

The differential conductance measurements of Fig. 4 cover the parameter region adjacent to the bandgap towards positive gate voltage, where few electrons are trapped in the carbon nanotube. Fig. 4(a) shows the $1 \leq N_{\mathrm{el}} \leq 8$ region in logarithmic color scale. The diamond-shaped regions of Coulomb
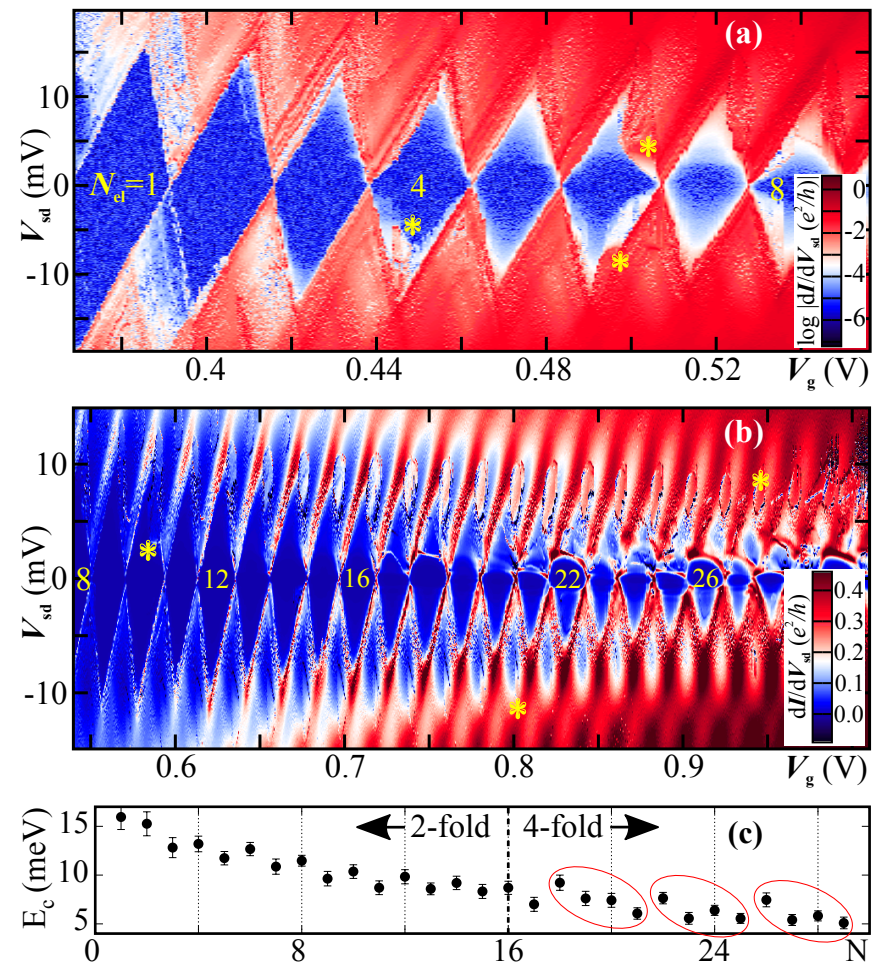

FIG. 4. Stability diagrams showing $\mathrm{d} I / \mathrm{d} V_{\mathrm{sd}}\left(V_{\mathrm{g}}, V_{\mathrm{sd}}\right)$ of the strong Coulomb blockade parameter region at low electron number: (a) $1 \leq$ $N_{\mathrm{el}} \leq 8$, logarithmic color scale, and (b) $8 \leq N_{\mathrm{el}} \leq 30$, linear color scale. Yellow asterisks mark (some of the) mechanical instability features. (c) Charging energy as function of electron number $N_{\mathrm{el}}$.

blockade and fixed number $N_{\mathrm{el}}$ of trapped electrons are clearly visible. Additionally, we observe multiple conductance lines in the single electron tunneling regions corresponding to excited quantum states. Already in the Coulomb blockade region with $N_{\mathrm{el}}=4$, further lobe-shaped features appear, becoming much stronger at $N_{\mathrm{el}}=6$ (see the yellow asterisks in the figure). These again correspond to vibrational feedback phenomena typical for clean and suspended carbon nanotube devices at millikelvin temperatures [16, 22, 27, 28].

Fig. 4(b) continues the plot of Fig. 4(a) towards larger electron numbers $8 \leq N_{\mathrm{el}} \leq 30$, now in linear color scale. The lobe-shaped instability regions now occur repetitively for every Coulomb oscillation. In addition, near-horizontal features in Coulomb blockade, which can be associated with inelastic cotunneling [33] and the non-equilibrium Kondo effect $[6,25,36]$, become increasingly prominent.

A plot of the charging energy $E_{c}\left(N_{\mathrm{el}}\right)$ as function of electron number $N_{\mathrm{el}}$ is provided in Fig. 4(c). It displays the overall decrease of $E_{c}$ with $N_{\text {el }}$, typical for this type of device, in addition to "shell-effects" from subsequent filling of quantum levels analogous to shells in atomic physics. Interestingly, the shell-filling results in a two-fold pattern for $N_{\mathrm{el}}<16$, while for $N_{\mathrm{el}}>16$ a fourfold peak grouping with the largest charging energy at $4 n+2$ is observed. Electron-electron interaction effects are a likely cause of this phenomenon [7], requiring further analysis and potentially additional measurements 
in magnetic fields.

Figure 5 illustrates the attempt to resolve the transversal vibration resonance for decreasing electron numbers $N_{\mathrm{el}}$ all the way to the electronic band gap. Even though a comparatively large bias of $V_{\mathrm{sd}}=2 \mathrm{mV}$ (see Fig. 5(a)) is applied, the resonance peaks only remain detectable for $N_{\mathrm{el}} \geq 9$, as marked by an ellipsiod in Fig. 5(b). The peaks are far in the nonlinear response regime, displaying an abrupt edge similar to a Duffing oscillator response at increasing driving frequency. For $N_{\mathrm{el}}<9$ the resonant response becomes too small to be detected. To be able to trace the transversal vibration resonance frequency across the low electron number region, and specifically also the electronic band gap, a different detection scheme is required.

\section{LARGE ELECTRON NUMBERS}

With increasing positive gate voltage $V_{\mathrm{g}}$ and thereby increasing number of trapped electrons $N_{\mathrm{el}}$, higher order tunneling processes become dominant in the transport spectrum. This is immediately visible in the differential conductance plots of Figs. 6(a) and (b), where the Kondo effect and (to a lesser degree) elastic cotunneling increase the signal. While the conductance trace $\mathrm{d} I / \mathrm{d} V_{\mathrm{sd}}\left(V_{\mathrm{g}}\right)$ retains an overall fourfold repetitive pattern, corresponding to the fourfold occupation of each longitudinal momentum state in the nanotube confinement potential, the precise shape of the conductance oscillations continuously evolves towards Kondo ridges and suppressed Coulomb blockade.

An example stability diagram, plotting the differential conductance $\mathrm{d} I / \mathrm{d} V_{\mathrm{sd}}\left(V_{\mathrm{g}}, V_{\mathrm{sd}}\right)$ as function of gate voltage $V_{\mathrm{g}}$ and
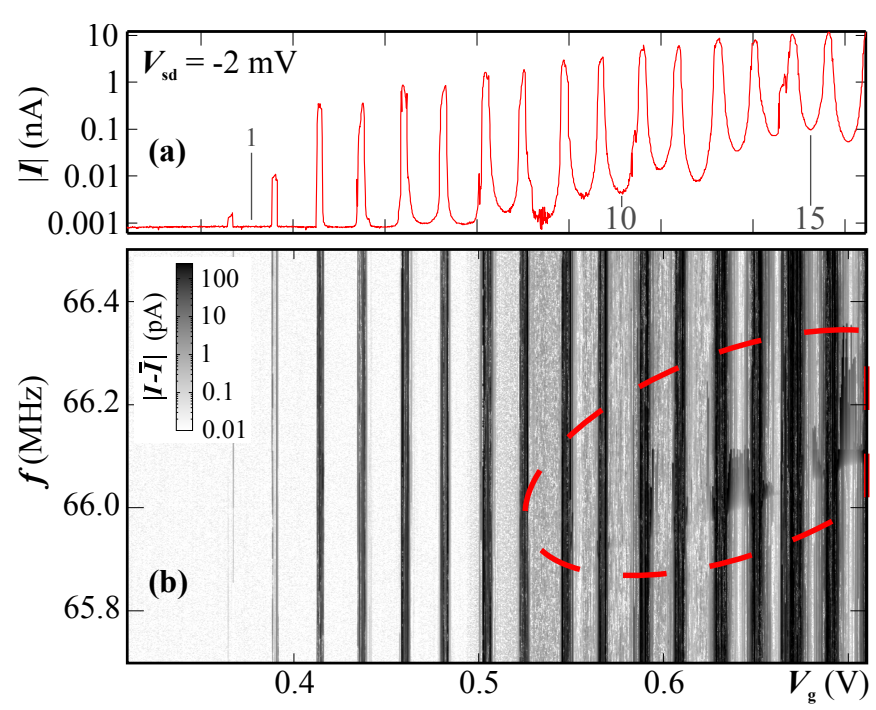

FIG. 5. (a) dc current $I\left(V_{\mathrm{g}}\right)$ at $V_{\mathrm{sd}}=-2 \mathrm{mV}$ for off-resonant $\mathrm{rf}$ driving signal; trace cut from the raw data of (b). (b) Mechanical resonance detection measurement at nominal $\mathrm{rf}$ power $P=-13.7 \mathrm{dBm}$; $\left|I\left(f, V_{\mathrm{g}}\right)-\bar{I}\left(V_{\mathrm{g}}\right)\right|$ in logarithmic scale. The resonance is indicated by a dashed ellipsoid.
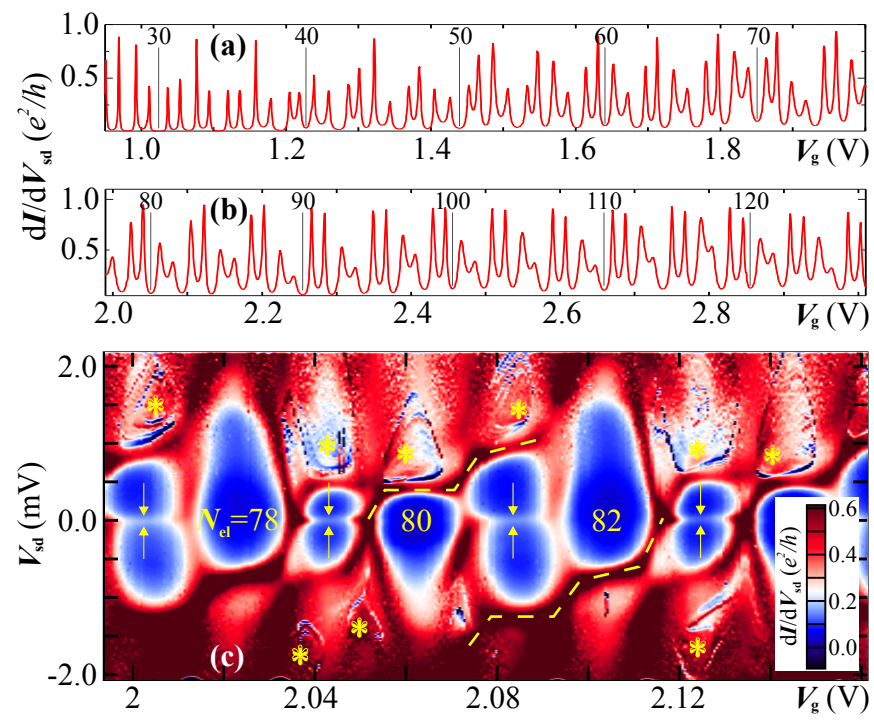

FIG. 6. (a), (b) Zero-bias differential conductance $\mathrm{d} I / \mathrm{d} V_{\mathrm{sd}}\left(V_{\mathrm{g}}\right)$ for increasingly positive gate voltage. The number of trapped electrons, $27 \leq N_{\mathrm{el}} \leq 77$ in (a) and $77 \leq N_{\mathrm{el}} \leq 128$ in (b), is indicated in the line plots. (c) Example stability diagram: differential conductance $\mathrm{d} I / \mathrm{d} V_{\mathrm{sd}}\left(V_{\mathrm{g}}, V_{\mathrm{sd}}\right)$ in the region $77 \leq N_{\mathrm{el}} \leq 85$; linear color scale, cut off at $0.6 e^{2} / h$ for better contrast.

bias voltage $V_{\text {sd }}$ in the region $77 \leq N_{\mathrm{el}} \leq 85$, is shown in Fig. 6(c). A zero bias conductance anomaly becomes clearly visible at odd electron number, though it is here still comparatively weak. An evaluation of the Kondo ridge width, following [37], leads to typical Kondo temperatures of $1 \mathrm{~K} \leq T_{\mathrm{K}} \leq$ $2 \mathrm{~K}$ at the center of the depicted odd $-N_{\mathrm{el}}$ Coulomb blockade regions.

Much more dominant in Fig. 6(c) are, however, different effects. As marked by dashed lines in the figure, strong, discrete conductance resonances at finite bias pass stepwise through the Coulomb blockade regions. Going beyond inelastic cotunneling, which would lead to a conductance threshold alone, these lines again correspond to nonequilibrium Kondo phenomena $[6,25,36]$. Similar "stepwise" resonance structures have been observed before and identified with an imbalance of the two tunnel barriers connecting a quantum dot to its leads [38]. Finally, regions of mechanical instability, marked with asterisks in Fig. 6(c), now consistently occur at nearly every electron number for bias values above $\left|V_{\mathrm{sd}}\right| \simeq 0.5 \mathrm{mV}$ and strongly distort the transport spectrum.

\section{CONCLUSIONS}

While a clean and suspended carbon nanotube may seem like a comparatively simple object, its spectroscopy reveals an immense richness of phenomena in electronics, nanomechanics, and the coupling of both. The tunability of the tunnel couplings via the gate voltage allows access to a wide parameter range, from the electronic band gap and strong Coulomb blockade all the way to higher order tunneling phe- 
nomena and eventually Fabry-Pérot interference. In nearly all of these regions nano-electromechanical phenomena can be identified. A driving signal makes it possible to detect the transversal vibration resonance frequency from low electron numbers all the way to the strong Kondo regime; even where charge quantization is nearly lost, a detailed analysis of the resonance frequency evolution still allows to characterize the electronic system via its quantum capacitance. Additionally, nano-electromechanical feedback effects dominate the current at finite bias, leading to strongly distorted transport spectra. The tunability of a single, clean device allows the direct comparison of parameter regimes [39], at known electron number and unchanged molecular structure.

\section{ACKNOWLEDGMENTS}

We would like to thank Ch. Strunk and D. Weiss for the use of experimental facilities. The authors acknowledge funding by the Deutsche Forschungsgemeinschaft via Emmy Noether grant $\mathrm{Hu}$ 1808/1, SFB 689, and SFB 1277. The measurement data has been recorded using the Lab::Measurement software package [40].

[1] E. A. Laird, F. Kuemmeth, G. A. Steele, K. Grove-Rasmussen, J. Nygård, K. Flensberg, and L. P. Kouwenhoven, "Quantum transport in carbon nanotubes," Rev. Mod. Phys. 87, 703 (2015).

[2] J. Cao, Q. Wang, and H. Dai, "Electron transport in very clean, as-grown suspended carbon nanotubes," Nature Materials 4, 745 (2005).

[3] F. Kuemmeth, S. Ilani, D. C. Ralph, and P. L. McEuen, "Coupling of spin and orbital motion of electrons in carbon nanotubes," Nature 452, 448 (2008).

[4] G. A. Steele, G. Gotz, and L. P. Kouwenhoven, "Tunable fewelectron double quantum dots and klein tunnelling in ultraclean carbon nanotubes," Nat. Nanotech. 4, 363 (2009).

[5] F. Pei, E. A. Laird, G. A. Steele, and L. P. Kouwenhoven, "Valley-spin blockade and spin resonance in carbon nanotubes," Nat. Nanotechn. 7, 630 (2012).

[6] D. R. Schmid, S. Smirnov, M. Margańska, A. Dirnaichner, P. L. Stiller, M. Grifoni, A. K. Hüttel, and Ch. Strunk, "Broken SU(4) symmetry in a Kondo-correlated carbon nanotube," Phys. Rev. B 91, 155435 (2015).

[7] V. V. Deshpande and M. Bockrath, "The one-dimensional Wigner crystal in carbon nanotubes," Nature Physics 4, 314 (2008).

[8] A. Hamo, A. Benyamini, I. Shapir, I. Khivrich, J. Waissman, K. Kaasbjerg, Y. Oreg, F. von Oppen, and S. Ilani, "Electron attraction mediated by Coulomb repulsion," Nature 535, 395 (2016).

[9] I. Shapir, A. Hamo, S. Pecker, C. Pascu Moca, Ö. Legeza, G. Zarand, and S. Ilani, "Imaging the Wigner crystal of electrons in one dimension," (2018), arXiv:1803.08523.

[10] J. O. Island, M. Ostermann, L. Aspitarte, E. D. Minot, D. Varsano, E. Molinari, M. Rontani, and G. A. Steele, "Interaction-driven giant orbital magnetic moments in carbon nanotubes,” Phys. Rev. Lett. 121, 127704 (2018).
[11] V. Sazonova, Y. Yaish, H. Üstünel, D. Roundy, T. A. Arias, and P. L. McEuen, "A tunable carbon nanotube electromechanical oscillator,” Nature 431, 284 (2004).

[12] B. Witkamp, M. Poot, and H.S.J. van der Zant, "Bendingmode vibration of a suspended nanotube resonator," Nano Lett. 6, 2904 (2006).

[13] A. K. Hüttel, G. A. Steele, B. Witkamp, M. Poot, L. P. Kouwenhoven, and H. S. J. van der Zant, "Carbon nanotubes as ultrahigh quality factor mechanical resonators," Nano Letters $\mathbf{9}$, 2547 (2009).

[14] E. A. Laird, F. Pei, W. Tang, G. A. Steele, and L. P. Kouwenhoven, "A high quality factor carbon nanotube mechanical resonator at $39 \mathrm{GHz}$," Nano Letters 12, 193 (2012).

[15] J. Moser, A. Eichler, J. Güttinger, M. I. Dykman, and A. Bachtold, "Nanotube mechanical resonators with quality factors of up to 5 million," Nature Nanotechnology 9, 1007 (2014).

[16] G. A. Steele, A. K. Hüttel, B. Witkamp, M. Poot, H. B. Meerwaldt, L. P. Kouwenhoven, and H. S. J. van der Zant, "Strong coupling between single-electron tunneling and nanomechanical motion," Science 325, 1103 (2009).

[17] A. K. Hüttel, H. B. Meerwaldt, G. A. Steele, M. Poot, B. Witkamp, L. P. Kouwenhoven, and H. S. J. van der Zant, "Single electron tunnelling through high-Q single-wall carbon nanotube NEMS resonators," Phys. Stat. Sol. b 247, 2974 (2010).

[18] B. Lassagne, Y. Tarakanov, J. Kinaret, D. Garcia-Sanchez, and A. Bachtold, "Coupling mechanics to charge transport in carbon nanotube mechanical resonators," Science 325, 1107 (2009).

[19] J. Kong, H. T. Soh, A. M. Cassell, C. F. Quate, and H. Dai, "Synthesis of individual single-walled carbon nanotubes on patterned silicon wafers," Nature 395, 878 (1998).

[20] J. Park and P. L. McEuen, "Formation of a p-type quantum dot at the end of an n-type carbon nanotube," Applied Physics Letters 79, 1363 (2001).

[21] M. Margańska, D. R. Schmid, P. L. Stiller, A. Dirnaichner, Ch. Strunk, M. Grifoni, and A. K. Hüttel, "Shaping electron wave functions in a carbon nanotube with a parallel magnetic field," (2017), arXiv:1712.08545.

[22] D. R. Schmid, P. L. Stiller, Ch. Strunk, and A. K. Hüttel, "Magnetic damping of a carbon nanotube nano-electromechanical resonator," New Journal of Physics 14, 083024 (2012).

[23] M. Poot, B. Witkamp, M. A. Otte, and H. S. J. van der Zant, "Modelling suspended carbon nanotube resonators," Phys. Stat. Sol. b 244, 4252 (2007).

[24] D. Goldhaber-Gordon, Hadas Shtrikman, D. Mahalu, David Abusch-Magder, U. Meirav, and M. A. Kastner, "Kondo effect in a single-electron transistor," Nature 391, 156 (1998).

[25] J. Nygård, H. C. Cobden, and P. E. Lindelof, "Kondo physics in carbon nanotubes," Nature 408, 342 (2000).

[26] P. Jarillo-Herrero, J. Kong, H. S. J. van der Zant, C. Dekker, L. P. Kouwenhoven, and S. De Franceschi, "Orbital Kondo effect in carbon nanotubes," Nature 434, 484 (2005).

[27] O. Usmani, Ya. M. Blanter, and Y. V. Nazarov, "Strong feedback and current noise in nanoelectromechanical systems," Phys. Rev. B 75, 195312 (2007).

[28] D. R. Schmid, P. L. Stiller, Ch. Strunk, and A. K. Hüttel, "Liquid-induced damping of mechanical feedback effects in single electron tunneling through a suspended carbon nanotube," Appl. Phys. Lett. 107, 123110 (2015).

[29] K. J. G. Götz, D. R. Schmid, F. J. Schupp, P. L. Stiller, Ch. Strunk, and A. K. Hüttel, "Nanomechanical characterization of the Kondo charge dynamics in a carbon nanotube," Phys. Rev. Lett. 120, 246802 (2018). 
[30] H. B. Meerwaldt, G. Labadze, B. H. Schneider, A. Taspinar, Y. M. Blanter, H. S. J. van der Zant, and G. A. Steele, "Probing the charge of a quantum dot with a nanomechanical resonator," Phys. Rev. B 86, 115454 (2012).

[31] W. Liang, M. Bockrath, D. Bozovic, J. H. Hafner, M. Tinkham, and H. Park, "Fabry-Pérot interference in a nanotube electron waveguide," Nature 411, 665 (2001).

[32] A. Dirnaichner, M. del Valle, K. J. G. Götz, F. J. Schupp, N. Paradiso, M. Grifoni, Ch. Strunk, and A. K. Hüttel, "Secondary electron interference from trigonal warping in clean carbon nanotubes," Phys. Rev. Lett. 117, 166804 (2016).

[33] S. De Franceschi, S. Sasaki, J. M. Elzerman, W. G. van der Wiel, S. Tarucha, and L. P. Kouwenhoven, "Electron cotunneling in a semiconductor quantum dot," Phys. Rev. Lett. 86, 878 (2001).

[34] A. Benyamini, A. Hamo, S. V. Kusminskiy, F. von Oppen, and S. Ilani, "Real-space tailoring of the electron-phonon coupling in ultraclean nanotube mechanical resonators," Nat. Phys. 10, 151 (2014).

[35] P. Häkkinen, A. Isacsson, A. Savin, J. Sulkko, and P. Hakonen, "Charge sensitivity enhancement via mechanical oscillation in suspended carbon nanotube devices," Nano Letters 15,
1667 (2015).

[36] M. Gaass, A. K. Hüttel, K. Kang, I. Weymann, J. von Delft, and Ch. Strunk, "Universality of the Kondo effect in quantum dots with ferromagnetic leads," Phys. Rev. Lett. 107, 176808 (2011).

[37] A. V. Kretinin, H. Shtrikman, and D. Mahalu, "Universal line shape of the Kondo zero-bias anomaly in a quantum dot," Phys. Rev. B 85, 201301 (2012).

[38] A. Makarovski, J. Liu, and G. Finkelstein, "Evolution of transport regimes in carbon nanotube quantum dots," Phys. Rev. Lett. 99, 066801 (2007).

[39] M. Niklas, S. Smirnov, D. Mantelli, M. Marganska, N.-V. Nguyen, W. Wernsdorfer, J.-P. Cleuziou, and M. Grifoni, "Blocking transport resonances via Kondo many-body entanglement in quantum dots," Nat. Commun. 7, 12442 (2016).

[40] S. Reinhardt, C. Butschkow, S. Geissler, A. Dirnaichner, F. Olbrich, C. Lane, D. Schröer, and A. K. Hüttel, "Lab::Measurement - a portable and extensible framework for controlling lab equipment and conducting measurements," Computer Physics Communications 234, 216 (2019). 